\title{
First-principles calculation of field evaporation and surface diffusion on BCC Fe (001)
}

Toshiharu Ohnuma ${ }^{1}$

1. Material Science Research Laboratory, Central Research Institute of Electric Power Industry (CRIEPI), 2-11-1 Iwado Kita, Komaeshi, Tokyo 201-8511, Japan

Atom probe tomography (APT) is a very powerful tool which can investigate atomic positions and chemical compositions of materials with sub nanometer resolution. In APT, atoms are field-evaporated by applying a high electric field to needle-like specimen[1]. At the precipitates and interfaces, due to the difference in the electric field evaporation voltage of the element, the local magnification effect causes artifacts[2]. Surface diffusion and reconstruction of atoms during field evaporation also causes $\operatorname{artifacts[3].~}$

The difference of the electric field evaporation voltage by the element can be obtained by first-principles calculation. As a method of applying an electric field in the first-principles calculation, a sawtooth electric field has been used in the three-dimensional periodic boundary condition, but this causes a difficulty of convergence of the electronic state. In recent years, an effective screen medium (ESM) method has been developed, and it becomes possible to perform a simulation in which the periodic boundary in the electric field direction is removed[4]. In this research, we perform first-principles calculation of surface diffusion and electric field evaporation where electric field is applied by ESM method.

The PWSCF code of Quantum Espresso package and a combination of the generalized gradient approximation (GGA) for the exchange and correlation energies and density functional theory were employed in this study[5,6]. We used the ultrasoft pseudopotential method[7]. We used a slab model based on a $4 \times 4 \times 4$ BCC lattice with a total of 128 atoms in the supercell. The both sides of the $\mathrm{Fe}(001)$ were assumed to be vacuum layers with a length exceeding $1.3 \mathrm{~nm}$. Brillouin sampling was performed by the Monkhorst-Pack scheme with a $2 \times 2 \times 1 \mathrm{mesh}[8]$. The cutoff energy of the plane waves was $25 \mathrm{Ry}$. The supercell used for calculation is shown in Fig 1.

In the field evaporation calculation of $\mathrm{Fe}$ from $\mathrm{Fe}$ (001), only the $\mathrm{z}$ direction of the adsorbed atoms was fixed, and the structure optimization of the surface and slab structure was carried out with the electric field of $0 \mathrm{~V} / \mathrm{nm}$. For the adsorbed Fe atoms, the position from the surface was changed to calculate the structure of 10 points. Figure 2 shows the potential energies in the Fe adsorption on Fe (001) when the electric fields are changed.

In the ESM method, a positive electric field is applied by removing charge from the Fe atoms of the slab. Calculations were performed for 12 cases with varying the electric field. The dependence of the activation energy by the electric field is shown in Fig 3. The field evaporation voltage of Fe obtained by the calculation was in good agreement with the experimental value. The field evaporation voltage of $\mathrm{Cu}$ from $\mathrm{Fe}(001)$ was almost the same as $\mathrm{Fe}$ from $\mathrm{Fe}(001)$.

Nudged Elastic Band (NEB) method was used to calculate the migration energy of surface diffusion by the electric field applied system. In order to calculate with constant electric field in the intermediate 
configuration of diffusion, it is necessary to control the Fermi level so that the electric field becomes constant (Constant- $\mu$ method), but it was difficult to converge by the conventional method. This can be solved efficiently by ESM method[9]. The surface migration calculations of Fe and $\mathrm{Cu}$ on $\mathrm{Fe}(001)$ were performed. As the electric field increased, the activation energy of diffusion decreased and the activation energy of the electric field of $28 \mathrm{~V} / \mathrm{nm}$ is about $40 \%$ of that of $0 \mathrm{~V} / \mathrm{nm}$.

References:

[1] M. K. Miller and R. G. Forbes, Atom-Probe Tomography: The Local Electrode Atom Probe, Springer (2014)

[2] C. Oberdorfer, G. Schmitz, Microscopy and Microanalysis 17 (2011) 15

[3] M. D. Mulholland and D. N. Seidman, Microscopy and Microanalysis 17 (2011) 950

[4] M. Otani and O. Sugino, Phys. Rev. B 73, (2006) 115407

[5] P. Giannozzi, S. Baroni, N. Bonini, M. Calandra, R. Car, C. Cavazzoni, D. Ceresoli, G. L. Chiarotti, M. Cococcioni, I. Dabo, A. Dal Corso, S. Fabris, G. Fratesi, S. de Gironcoli, R. Gebauer, U. Gerstmann, C. Gougoussis, A. Kokalj, M. Lazzeri, L. Martin-Samos, N. Marzari, F. Mauri, R. Mazzarello, S. Paolini, A. Pasquarello, L. Paulatto, C. Sbraccia, S. Scandolo, G. Sclauzero, A. P. Seitsonen, A. Smogunov, P. Umari, R. M. Wentzcovitch, J.Phys.:Condens.Matter 21, 395502 (2009), http://arxiv.org/abs/0906.2569 [6] P. Perdew, K. Burke, and M. Ernzerhof, Phys. Rev. Lett. 77, 3865 (1996)

[7] We used the pseudopotentials Fe.pbe-nd-rrkjus.UPF and Cu.pbe-d-rrkjus.UPF from http://www.quantum-espresso.org

[8] H. J. Monkhorst and J. D. Pack, Phys. Rev. B13, 51888 (1976)

[9] N. Bonnet, T. Morishita, O. Sugino, M. Otani, Phys. Rev. Lett. 109, (2012) 266101

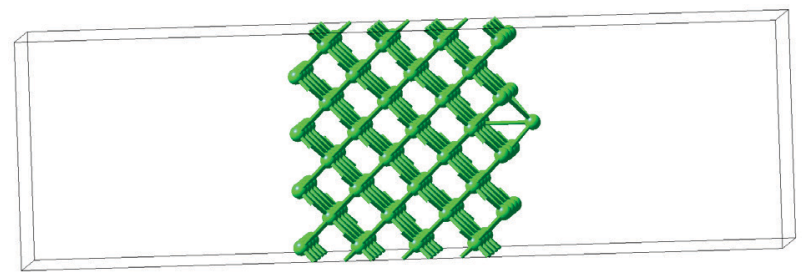

Fig.1 Supercell for calculation of Fe on $\mathrm{Fe}(001)$.

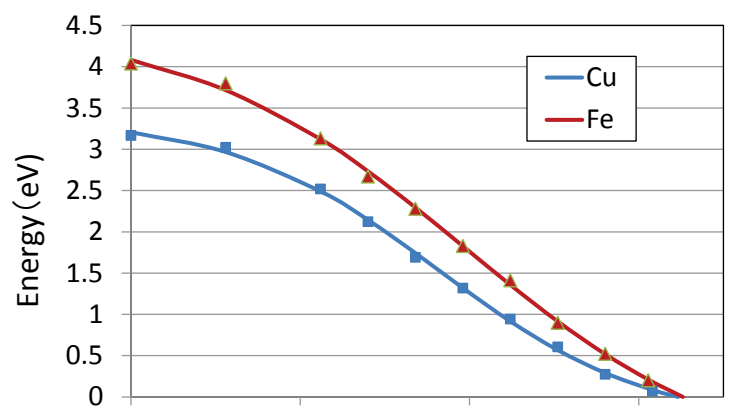

Fig. 3 The dependence of the activation energy by the electric field for $\mathrm{Fe}$ and $\mathrm{Cu}$ on $\mathrm{Fe}(001)$.

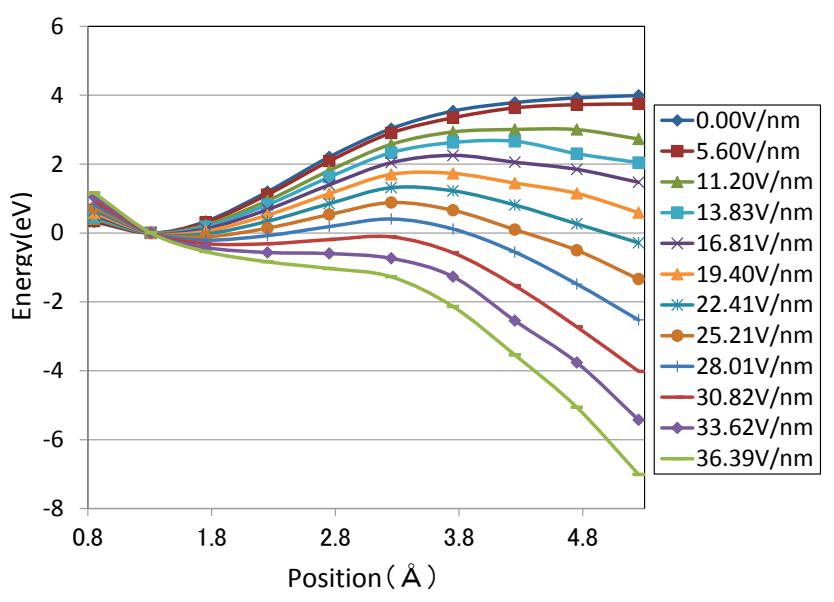

Fig.2 Energy of the system as a function of an ad-atom displacement for $\mathrm{Fe}$ on $\mathrm{Fe}(001)$. 